(2) Open Access Full Text Article

REVIEW

\title{
Drug targets and predictive biomarkers in the management of metastatic melanoma
}

This article was published in the following Dove Press journal:

Pharmacogenomics and Personalized Medicine

27 September 2012

Number of times this article has been viewed

\author{
Jaykumar Thumar \\ Eva Giesen \\ Harriet $M$ Kluger \\ Yale Cancer Center, Yale School \\ of Medicine, New Haven, CT, USA
}

Correspondence: Harriet Kluger

Yale Cancer Center, Yale School

of Medicine, 333 Cedar Street,

WWW2I 3 New Haven, CT 0650I, USA

$\mathrm{Tel}+$ I 203785622 I

Fax +I 2037853788

Email harriet.kluger@yale.edu

\begin{abstract}
Melanoma is the leading cause of fatal skin cancer, and in the past few decades, there has been an increase in the incidence of and mortality from metastatic melanoma. Until recently, the therapeutic options for treatment of metastatic melanoma were limited. The approval of ipilimumab (an anti-CTLA-4 antibody) and vemurafenib (mutant B-RAF ${ }^{\mathrm{V} 600 \mathrm{E}}$ kinase inhibitor) by the Federal Drug Administration has led to a new era in melanoma treatment, and additional promising drugs and drug combinations are currently being investigated. As the choices of treatment for melanoma have expanded, the need to identify predictive biomarkers to tailor treatment strategies to individual tumor or immune system characteristics has become necessary. Such strategies have the potential of maximizing antitumor effect while minimizing toxicity and improving clinical benefit. In this article, we review the currently approved targeted therapies in melanoma and discuss the future of personalized therapy for this disease.
\end{abstract}

Keywords: vemurafenib, mutations, inhibitors, tumors

\section{Introduction}

In the United States, melanoma is the fifth-leading cancer in men and the seventh in women. In recent years, the incidence of melanoma has increased, and despite improvements in awareness and early detection, the mortality from metastatic melanoma is on the rise, particularly in elderly patients. ${ }^{1}$ The prognosis of patients with metastatic melanoma remains extremely poor, with the median survival ranging from 8 to 18 months after diagnosis. $^{2}$ Until 2011, only two therapies (dacarbazine, [Bayer- Hospira Inc. Lake Forest, IL (DTIC)] and high-dose interleukin-2 [Prometheus labs, San Diego, CA (IL-2)]) had been approved for metastatic melanoma by the Federal Drug Administration (FDA); both of these agents have not been shown to improve overall survival. ${ }^{3,4}$ Two recently approved therapies, ipilimumab (Bristol-Myers Squibb, Princeton, NJ) (anti-CTLA-4 antibody, an immune therapy) and vemurafenib (Genentech, South San Francisco, CA) (B-RAF ${ }^{\mathrm{V} 600 \mathrm{E}}$ kinase inhibitor), have shown a survival benefit in large randomized clinical trials. A large number of therapeutic agents currently in clinical development are likely to expand the therapeutic options for melanoma.

\section{Personalizing immune therapies}

The response rates (RRs) for the currently available immune therapies (IL-2 and ipilimumab) are low, while experimental agents have been associated with higher RRs in small nonrandomized trials. ${ }^{3,5-7}$ The objective RR for high-dose IL-2 for selected patients with a good performance status is approximately $15 \%$, including durable, long-term 
complete responses (CRs) in approximately 5\% of all treated patients. ${ }^{3,6}$ Similarly lower RRs are seen with ipilimumab $(\sim 15 \%)$, while RRs to investigational agents such as anti-PD-1 (Bristol-Myers Squibb, Princeton, NJ) appear to be somewhat higher $(-30 \%) .{ }^{5}$ Adoptive cell therapy is a treatment that uses $\mathrm{T}$ cells harvested from tumor-infiltrating lymphocytes (TILs) from resected tumors or peripheral blood activated and expanded. These tumor antigen-specific lymphocytes are expanded ex vivo and reinfused after partial or complete myeloablation. ${ }^{8}$ Adoptive cell therapy results in higher RRs (approximately $50 \%$ ) in highly selected patients in Phase I trials; however, this modality of therapy is available only at select centers, and larger/randomized studies are difficult to conduct. ${ }^{8}$

Despite the low RR for immune therapies, clinical experience indicates that small subsets of patients achieve apparent durable benefit, and can potentially be cured. Thus identifying predictive markers could have a tremendous impact on personalizing immunotherapy in melanoma by enabling selection of treatment for patients who are more likely to respond. However, due to the complexity of interactions between tumors and the immune system that affect antitumor responses, discovery of such biomarkers has been particularly challenging.

\section{Predicting response to ipilimumab}

Ipilimumab is the first immunotherapeutic agent to improve overall survival in metastatic melanoma, as demonstrated in two randomized Phase III clinical trials that led to its approval by the FDA in March 2011. ${ }^{9,10}$ Although ipilimumab is associated with longer overall survival, the RR (CR and partial response [PR]) was only $10 \%-15 \%$, and the rate of CR, PR, and stable disease (SD) in combination was approximately 30\%. Given that the majority of patients do not respond to this drug, and given the associated toxicities, incorporation of predictive biomarkers could potentially improve the therapeutic ratio of ipilimumab and are the subject of intensive research. ${ }^{11}$

Expression of FOXP3 and indoleamine 2,3-dioxygenase (IDO) in the tumor microenvironment has been shown to be associated with clinical activity in patients treated with ipilimumab. ${ }^{12}$ Among 82 patients treated with ipilimumab, pretreatment samples stained for FOXP3 and IDO revealed higher RRs for patients whose tumors expressed high levels of IDO ( $\mathrm{n}=35)$ and FOXP3 $(\mathrm{n}=33)$; for high and low expression, RRs for IDO and FOXP3 were $40 \%$ vs $11 \%(P=0.014)$ and $50 \%$ vs $10 \%(P=0.012)$, respectively. Analysis of the TILs within the tumor tissues showed that the increased density of the TILs 3 weeks after the start of treatment was associated with improved clinical benefit $(P=0.005) .{ }^{13}$
Recently, Yuan et al showed that high titers of pretreatment anti-NY-ESO-1 antibodies can predict response to ipilimumab. ${ }^{14}$ NY-ESO-1 seropositivity predicted improved clinical benefit, defined as the combination of $\mathrm{CR}, \mathrm{PR}$, and SD $(P=0.02)$. Furthermore, analysis of NY-ESO-1specific $\mathrm{CD}^{+}$and $\mathrm{CD}^{+}$T-cell responses by intracellular multicytokine staining revealed that patients with pretreatment anti-NY-ESO-1 antibodies who developed $\mathrm{CD}^{+} \mathrm{T}$-cell responses were more likely to respond to the drug treatment (10 of $13 ; 77 \%$ ) than those with undetectable $\mathrm{CD}^{+} \mathrm{T}$-cell responses (one of seven; $14 \% ; P=0.02$, relative risk $=5.4$ ), and were more likely to live longer $(P=0.01)$. However, an attempt to reproduce these results was unsuccessful; a retrospective analysis of patients treated with ipilimumab at the Surgery Branch of the National Institutes of Health failed to show a correlation between pretreatment or posttreatment seropositivity to NY-ESO-1 and response to ipilimumab ( $P=1.0$ and $P=0.7$, respectively). ${ }^{15}$ Since the results for these two retrospective reports are conflicting, further studies with larger patient cohorts are required to test the validity of NY-ESO-1 antibodies as predictive biomarkers of response to ipilimumab.

Ongoing studies are investigating additional biomarkers in melanoma patients receiving ipilimumab. ${ }^{16}$ A large intergroup study (NCT01489423) is investigating various blood and tissue biomarkers, such as circulating immune effector cells (T, B, NK, and NK-T cells), circulating plasmacytoid dendritic cells, myeloid dendritic cells, and melanoma-associated antigen-specific $\mathrm{T}$ cells as predictors of the response to ipilimumab. ${ }^{17}$

\section{Predicting response to anti-PD-I antibodies}

Antiprogrammed death 1 (PD-1) antibodies have shown promising results in early Phase clinical trials. PD-1, a receptor expressed on $\mathrm{T}$ cells, is an important negative immune checkpoint molecule that inhibits activation of cytotoxic T lymphocytes. Tumor cells and stromal cells can express PD-1 ligands PD-L1 (B7-H1) and PD-L2 (B7-DC), and thus suppress T-cell activation. ${ }^{18,19}$ In preclinical studies, inhibition of the interaction between PD-1 and PD-L1 has been shown to enhance T-cell responses and antitumor activity. ${ }^{20,21}$

In a Phase I/II clinical trial with the anti-PD-1 antibody BMS-936558, a response rate of $28 \%$ was seen among melanoma patients at doses ranging from 0.1 to $10.0 \mathrm{mg} / \mathrm{kg}$ intravenously. ${ }^{18}$ Stable disease lasting 24 weeks or more was observed in additional patients. BMS-936558 was well 
tolerated overall, but was associated with immune-related adverse events, including pneumonitis, vitiligo, colitis, hepatitis, hypophysitis, and thyroiditis. ${ }^{18,19}$ There were three drug-related deaths (1\%) due to pneumonitis. ${ }^{18}$ The expression of PD-L1 by immunohistochemistry on pretreatment tumor samples of 42 patients was associated with a greater likelihood of response to treatment. Nine of 25 patients with PD-L1positive tumors responded, while no responses were seen among the 17 patients whose tumors did not express PD-L1 $(P=0.006)$, suggesting that PD-L1 in tumor tissue could serve as a predictive biomarker. ${ }^{18}$ Prospective validation is needed.
In addition, studies of antibodies to PL-L1 are ongoing (see www.clinicaltrials.gov).

\section{Melanocyte drug targets} The MAPK pathway in melanoma (Figure I)

The growing understanding of the biology and pathogenesis of melanoma has resulted in a path of development of targeted therapies, which has led to initial improvement in the care of subsets of patients with advanced melanoma. One of the most significant discoveries in the field of melanoma

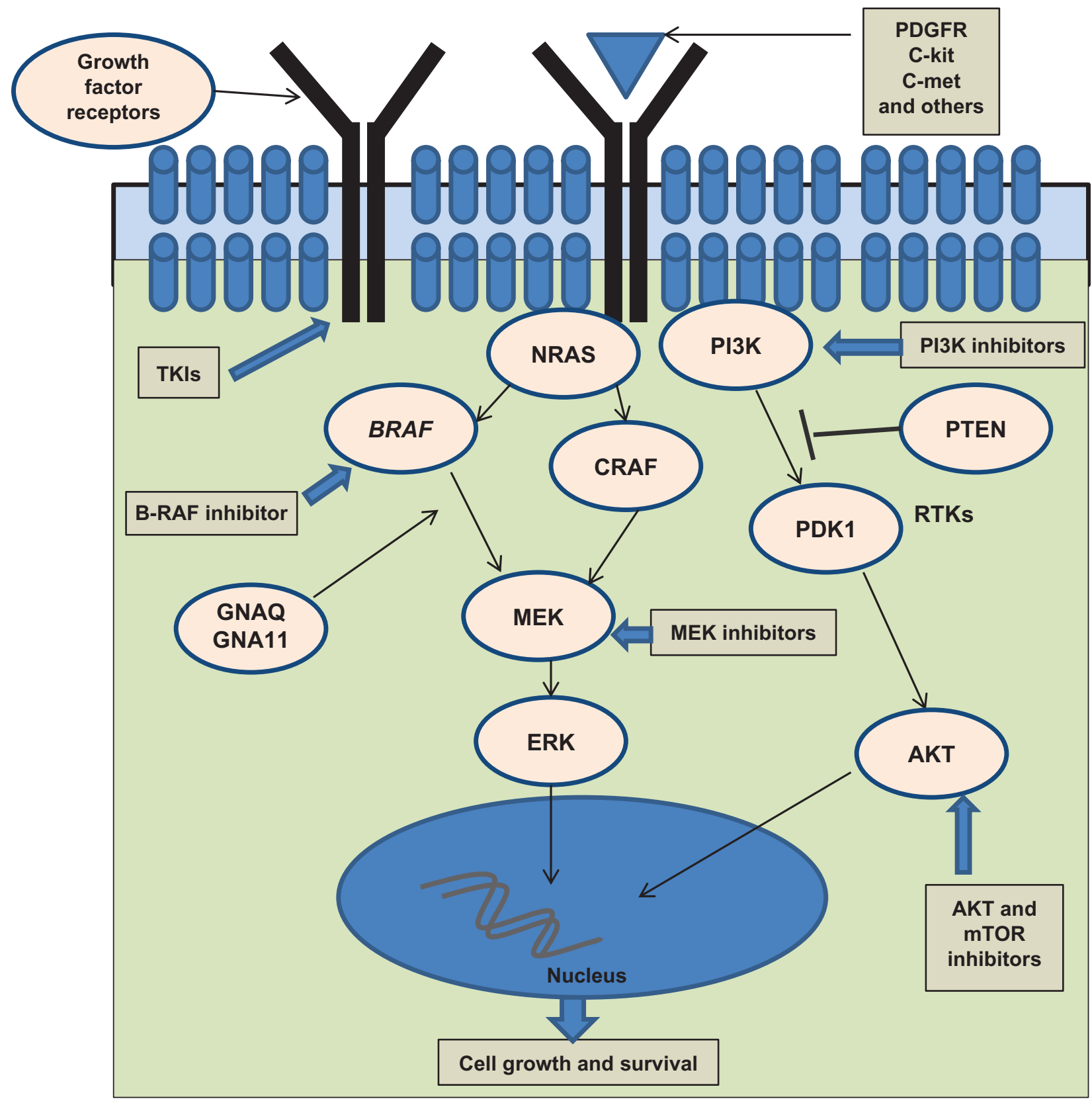

Figure I Cell-signaling pathways in melanoma. 
in recent years was the elucidation of the role of mitogenactivated protein kinase (MAPK) pathway, particularly the roles of mutant B-RAF and N-RAS.

The RAS-RAF-MAPK signaling pathway is activated in the vast majority of melanomas. In nonmalignant cells, the binding of growth factor receptors (such as epidermal growth factor receptor, c-Met, and c-KIT) to their corresponding ligand activates this intracellular kinase cascade. The MAPK pathway is activated in human melanomas either due to increased growth factor signaling or by genetic alterations in RAS and RAF proteins. ${ }^{22}$ MAPK regulates the activities of several transcription factors, such as C-Myc, CREB, and C-Fos. By altering the levels and activity of transcription factors, MAPK leads to altered transcription of genes that are fundamental for cell division and survival. The activation of B-RAF and the downstream signaling is also associated with enhanced NF $\kappa B$ promoter activity. ${ }^{23}$ Inhibition of B-RAF signaling has been shown to decrease NFאB promoter activity associated with cell survival, invasiveness, and angiogenesis. ${ }^{23,24}$

Vemurafenib (PLX4032 [Genentech, South San Francisco, $\mathrm{CA}$ ], Zelboraf), a B-RAF ${ }^{\mathrm{V} 600 \mathrm{E}}$ inhibitor with increased selectivity for mutant B-RAF ${ }^{\mathrm{V} 600 \mathrm{E}}$, was recently approved by the FDA for treatment of unresectable melanoma harboring B-RAF ${ }^{\mathrm{V} 60 \mathrm{E}}$ mutations. Dabrafenib (GlaxoSmithKline, Brentford, UK, GSK2118436), another specific inhibitor of mutant B-RAF ${ }^{\mathrm{V} 600 \mathrm{E}}$ kinase, has shown significant clinical efficacy in Phase II trials, and a Phase III clinical trial has completed accrual (NCT01245062). Additional molecules targeting the MAPK kinase pathway, including MEK inhibitors (trametinib [GlaxoSmithKline, Brentford, UK], MEK162 [Array Biopharma, Boulder, CO], TAK-733 [Millennium Pharmaceuticals, Inc., Cambridge, MA]), combinations of RAF and MEK inhibitors, pan-RAF inhibitors (such as RAF265), and others, have shown promising results in preclinical studies and are being investigated in clinical trials. These molecules, alone and in combination with additional inhibitors of the MAPK pathway or parallel pathways, hold the promise of expanding the therapeutic options for melanoma patients, and provide the first tools for personalized therapy for patients whose tumors harbor MAPK pathway-activating mutations.

\section{Mutations in $B-R A F$}

Mutations in the $B-R A F$ gene are found early in the development of melanoma, and can also be seen in benign nevi. The most common mutation of B-RAF ( $~ 80 \%)$ in melanoma is V600E and it involves substitution of valine to glutamic acid. ${ }^{25,26}$ Less common mutations are the V600K $(\sim 20 \%)$ and the V600D or V600R $(\sim 3 \%) .{ }^{27} B-R A F$ mutations are found in various categories of melanocytic growth, including melanocytic nevi (70\%-80\%), vertical growth-phase melanoma (40\%-50\%), and metastatic melanoma $(40 \%-50 \%) .{ }^{28}$ The precise role of $B-R A F$ mutations in oncogenesis is unclear, but resultant constitutive activation of the MAPK pathway causes cellular growth and vascular development in melanoma tumors. ${ }^{29}$

\section{Mutations in N-RAS}

The RAS family members are G proteins, which serve as critical mediators in the transduction of growth signals into the cell. $N-R A S$ mutations have been identified in $15 \%-20 \%$ of cutaneous melanoma and are presumed to be one of the important drivers of oncogenesis. ${ }^{30}$ A somatic mutation in the $N-R A S$ gene causes constitutive activation of the N-RAS protein, which leads to the successive activation of downstream serine/threonine kinases, which promote cell cycle progression, cellular transformation, and enhanced cell survival. ${ }^{30}$ The overexpression of growth factor receptors, such as epidermal growth factor receptor, c-Met, plateletderived growth factor receptor (PDGFR), fibroblast growth factor receptor, and c-KIT is also implicated as a mechanism of cellular growth and transformation in RAS-driven melanomas. ${ }^{30,31}$ The most important downstream mediators of activated RAS are the serine/threonine kinases B-RAF and C-RAF, which are activated following RAS binding.

The most frequent mutations in $N-R A S$ affect exon 2 (codon 60 and 61) and exon 1 (codon 12 and 13). Substitutions at positions 60 and 61 accounted for $82.4 \%$ of N-RAS mutations, most frequently a glutamine to arginine/lysine/ leucine substitution at position $61(\mathrm{Q} 61 \mathrm{R} / \mathrm{K} / \mathrm{L})$. Other frequent mutations (17.6\%) affect G12 and G13 on exon $1.32-34$

\section{Impact of $B-R A F$ and N-RAS mutations on clinicopathological profile, prognosis, and response to therapy}

$B-R A F$ and $N-R A S$ mutations appear to be biologically different than wild-type (WT) tumors and clearly have an impact on the clinical course. In early stage melanoma, $N-R A S$ mutations are associated with thicker tumors; $75 \%$ of $N-R A S$ mutant melanomas are $>1 \mathrm{~mm}$ in depth, while $40 \%$ of $B-R A F$ and $34 \%$ of WT primary melanomas are thicker than $1 \mathrm{~mm} . N-R A S$ mutations were also associated with increased proliferation rates, with $75 \%$ having $>1$ mitosis $/ \mathrm{mm}^{2}$, versus $40 \%$ for $B-R A F$ mutations and $55 \%$ for $\mathrm{WT}^{33}$ The majority of early stage melanomas with $B-R A F$ mutations are the superficial spreading subtype (88\%), and $B-R A F$ mutations are more likely to be found in body areas with intermittent sun exposure compared to chronic or no sun exposure $(P=0.02) .{ }^{35,36}$ 
$N-R A S$ mutation status has been identified as an independent predictor of shorter survival in patients with metastatic melanoma ( $N-R A S$ vs WT, 8.2 vs 15.1 months; $P=0.004){ }^{32}$ The risk of brain metastasis at the time of diagnosis of stage IV disease was also noted to be significantly higher in $B-R A F$ mutant (24\%) and $N-R A S$ mutant (23\%) patients compared with WT patients $(12 \%)$. However, patients with $B-R A F$ mutant melanoma treated with specific B-RAF inhibitors have an improved survival compared with $N-R A S$ and tumors that are WT for both. ${ }^{32,36} N-R A S$ mutation might be predictive of improved response to high-dose IL-2: a recent retrospective analysis showed differences in response to IL-2 in melanoma patients based on the mutation status (N-RAS 47\%, B-RAF 23\%, WT 12\%; P = 0.05). ${ }^{37}$ Patients with $N-R A S$ mutations had nonstatistically significant longer overall survival (5.3 vs 2.4 years; $P=0.30$ ) and progressionfree survival (214 vs 70 days; $P=0.13$ ).

\section{Other MAPK pathway mutations GNAQ and GNA I I mutations in uveal melanoma}

Activating somatic mutations in either of the homologous G-protein GNAQ or GNA11 genes lead to activation of MAPK kinase pathway in uveal melanoma. Mutations affecting Q209 in GNA11 were seen in 32\% of primary uveal melanomas and in $57 \%$ of uveal melanoma metastasis. ${ }^{38,39}$ The Q209 mutation in GNAQ was seen in $45 \%$ of the uveal melanoma and $22 \%$ of the metastatic tumors. Transduction of mutated GNA11 in murine models showed enhanced growth and metastasis in xenografts and also revealed activation of the MAPK pathway. ${ }^{40}$ The molecules mediating cross-talk between these mutant proteins and MAPK pathway remains elusive. Data derived from a limited number of patients suggest that these mutations do not have prognostic significance. ${ }^{40}$

\section{KIT mutations in acral lentiginous and mucosal melanoma}

C-KIT is a transmembrane receptor that belongs to the receptor tyrosine kinase family of proteins and is normally expressed on a restricted set of cell types, including melanocytes and interstitial cells of Cajal. ${ }^{41,42}$ The endogenous ligand for c-KIT is stem cell factor. Binding of stem cell factor to c-KIT results in activation of several intracellular signaling pathways involved in cell proliferation, invasion, metastasis, and inhibition of apoptosis. ${ }^{43}$ Several activating mutations in c-KIT have been described that result in ligand-independent constitutive activity of the tyrosine kinase.

Activating $c$-KIT mutations are commonly found in gastrointestinal stromal tumors, which are thought to originate from interstitial cells of Cajal. ${ }^{43}$ In addition, c-KIT mutations are frequently present in three subpopulations of melanoma: mucosal $(15 \%-27 \%)$, acral $(9 \%-23 \%)$, and less frequently in sun-damaged cutaneous melanomas $(0 \%-16 \%) .{ }^{44-46}$

\section{Molecular-targeted therapy in melanoma \\ B-RAF-targeted therapy in melanoma}

Development of drugs targeting mutant B-RAF ${ }^{\mathrm{V} 600 \mathrm{E}}$ is a major advance in personalized therapy for melanoma. The two agents that have demonstrated the most dramatic clinical benefit in melanoma are vemurafenib (PLX4032) and dabrafenib (GSK2118436).

\section{Vemurafenib}

A parental compound of vemurafenib, PLX4720, a 7-azaindole derivative, was discovered by using a structure-guided discovery approach, and therefore it preferentially inhibits the constitutively active mutant $\mathrm{B}-\mathrm{RAF}^{\mathrm{V} 600 \mathrm{E}}$ compared with other kinases, and potent cytotoxic effects are also exclusive to cells bearing the V600-mutated allele. ${ }^{47}$ In a multicenter, Phase I, dose-escalation trial of PLX4032, the maximum-tolerated oral dose (960 mg twice daily) was established..$^{48}$ In the expansion cohort, which consisted of patients with melanomas harboring B-RAF ${ }^{\mathrm{V} 600 \mathrm{E}}$ mutations, 26 of the 32 patients had objective responses $(81 \%)$, with a $\mathrm{CR}$ in two patients and a PR in 24 patients. ${ }^{48}$ B-RAF in Melanoma 2 (BRIM-2) was a Phase II study that enrolled 132 patients with previously treated stage IV melanoma with B-RAF ${ }^{\mathrm{V} 600 \mathrm{E}}$ mutations, and demonstrated an RR of $53 \%$, SD in $29 \%$, median progression-free survival (PFS) of 6.7 months, and an OS at 6 and 12 months of 77\% and $58 \%$, respectively. ${ }^{49}$

The efficacy of vemurafenib was confirmed in a Phase III trial, BRIM-3, that compared vemurafenib to DTIC in 675 previously untreated patients, whose tumors harbored B-RAF ${ }^{\mathrm{V} 600 \mathrm{E}}$ mutations. ${ }^{50}$ At interim analysis, the vemurafenib arm was associated with a significantly improved RR (48\% vs $4 \%, P<0.001$ ), median PFS (5.3 vs 1.6 months, hazard ratio $0.26,95 \% \mathrm{CI}: 0.20-0.33 ; P<0.001)$, and an improved OS at 6-month follow-up ( $84 \%$ vs $64 \%$, hazard ratio for death 0.37, 95\% CI: 0.26-0.55; $P<0.001)$.

\section{Dabrafenib}

This is a highly potent, selective ATP-competitive B-RAF inhibitor. In the preliminary results of a Phase I/II study, dabrafenib was shown to be very active in patients with metastatic melanoma, and a safe oral dose of $150 \mathrm{mg}$ twice daily was established. ${ }^{51}$ Among the 57 melanoma patients 
with $B-R A F$ mutations, a response rate of $27 \%$ was noted, including ten of 16 at the planned Phase II dose. A Phase III trial comparing dabrafenib with DTIC has completed accrual and final results are pending (NCT01227889). Dabrafenib also was noted to have activity against small, previously untreated brain metastases, and currently a Phase I/II trial is investigating its efficacy in central nervous system metastasis (NCT01266967).

\section{Toxicities of selective B-RAF inhibitors}

Overall, both vemurafenib and dabrafenib are well tolerated. Common adverse events associated with vemurafenib are arthralgia, rash, fatigue, alopecia, skin side effects, nausea, and diarrhea. Vemurafenib is associated with unexpected cutaneous toxicities that include squamous cell carcinomas of the skin $(12 \%)$, keratoacanthomas ( $8 \%)$, and photosensitivity $(30 \%)$. The reported incidence of non-melanoma skin cancer was lower $(\sim 2 \%)$ with dabrafenib in the Phase I/II trials. Molecular studies indicate that these lesions are due to paradoxical activation of the MAPK pathway that bypasses the inhibition of B-RAF. Furthermore, the short latency period until the development of these skin lesions is consistent with the presence of preexisting $R A S$ mutations in the skin that become oncogenic when subjected to B-RAF inhibition. ${ }^{52}$

\section{Testing for $B-R A F$ mutations in tumor samples}

Mutations in $B-R A F$ in tumor samples can be detected by a number of molecular methods. The Cobas 4800 B-RAF ${ }^{600}$ Mutation Test (LabCorp, Burlington, NC) is a PCR-based diagnostic test developed by Roche Molecular Systems (Pleasanton, CA) and was used to detect V600E mutations in tumor samples for patient selection for the BRIM-2 and BRIM-3 clinical trials. ${ }^{53}$ It was approved by the FDA as a companion diagnostic for vemurafenib.

There are various methods of sequencing DNA, and traditional direct (Sanger or dideoxy) sequencing is a commonly used method for mutation testing in clinical laboratories. However, the Sanger sequencing method, which uses sequence-specific termination of a DNA synthesis reaction using modified nucleotide substrates, suffers from limited sensitivity for detecting mutations that are present in low percentages in a tumor tissue..$^{54}$

The 454 method (massively parallel pyrosequencing method) was used as a gold standard to evaluate the discordances between polymerase chain reaction (Cobas) and Sanger results. The Cobas test was shown to be superior in detecting B-RAF ${ }^{\mathrm{V} 600 \mathrm{E}}$ mutations to Sanger sequencing. ${ }^{55}$ However, the sensitivity of this test to detect V600K $(10 \%-$
$20 \%$ of all $B-R A F$ ) is only $70 \%$, and additional testing might be needed to identify $\mathrm{V} 600 \mathrm{~K}$ mutations to avoid exclusion of patients who might benefit from B-RAF-targeting drugs. ${ }^{53}$ Detection of B-RAF mutation by immunohistochemistry using mutation-specific antibodies has shown remarkable specificity when confirmed by direct sequencing methods. This was studied on paraffin-embedded tissue samples of brain metastasis of various primary cancers $(50 \%$ of which were melanoma) and hairy cell leukemia, and future application of this test on a larger melanoma sample cohort is warranted to confirm the validity of this test. ${ }^{56,57}$

\section{Targeting c-KIT in melanoma}

Imatinib (Novartis, NY, NY) a multikinase inhibitor of c-KIT, PDGFR- $\alpha$ and $-\beta$, and Bcr-Abl, was successfully used in the treatment of chronic myelogenous leukemia and gastrointestinal stromal tumors. ${ }^{58,59}$ Given that melanoma cells express several protein tyrosine kinases, studies were initiated to assess the activity of imatinib in melanoma. ${ }^{60}$ Two trials failed to demonstrate clinical activity of imatinib in unselected melanoma patients. ${ }^{61,62}$ Importantly however, subsequent analysis of available melanoma cell lines showed no $c$-KIT mutations, ${ }^{61}$ and none of the analyzed melanomas demonstrated strong staining for C-KIT. ${ }^{62}$ In another study, imatinib treatment of patients whose melanomas expressed at least one of the target protein tyrosine kinases showed a PR in only one out of 21 patients. Interestingly, despite strong c-KIT staining by immunohistochemistry, $c-K I T$ mutation, copy number variation, and gene amplification could not be identified. An alternative splice variant was found in exon 15 , the significance of which was unclear. ${ }^{63}$ In contrast, case reports were subsequently published showing substantial responses to imatinib in melanomas harboring c-KIT mutations. ${ }^{64-67}$ This led to the initiation of several Phase II trials in which metastatic melanoma patients, with either amplification or mutation of $c-K I T$ in their tumors, were treated with imatinib. A Phase II trial by Guo et al in 2011 showed a promising 6-month PFS rate of $36.6 \%$ and a 1-year overall survival of $51.0 \%$. Partial responses were seen in $23 \%$ and tumor shrinkage in $41.9 \%$ of the 43 patients. Most of the responses were seen in tumors with $c$-KIT mutations in exons 11 and $13 .{ }^{68}$ In a second study of imatinib in patients with $c$-KIT mutations and/or amplifications, complete durable responses were seen in two out of 25 patients, while four patients achieved PRs (two durable), and prolonged stable disease ( $\geq 6$ months) was achieved in two patients. Similar to the previous study, most responders were found to have $c$-KIT mutations in exon 11 or exon $13 .{ }^{44}$ 
Although most published case reports and Phase II studies used imatinib as a $c-K I T$ inhibitor, clinical responses in patients with $c$-KIT mutant melanomas to treatment with other small molecule kinase inhibitors including dasatinib (BristolMyers Squibb, Princeton, NJ), sorafenib (Bayer Healthcare Pharmaceuticals, Inc, Leverkusen Germany and Onyx Pharm, Inc, South San Francisco, CA) nilotinib (Novartis, NY, NY), and sunitinib (Pfizer, NY, NY) have been reported. ${ }^{69-73}$

In summary, these studies indicate that treatment of melanoma with c-KIT inhibitors is only effective in the small subset of melanomas that harbor $c$-KIT mutations; melanoma tumors with strong c-KIT expression without mutation or amplification of the gene do not appear to respond to c-KIT inhibitors. Further characterization of the $c$-KIT mutations in relation to response to c-KIT inhibitors is needed, since early evidence suggests that mutations in exons 11 and 13 (ie, L576P and K642E) are more likely to respond to therapy.

\section{MEK inhibitors in melanoma}

MEK is an attractive therapeutic target since MEK inhibitors have shown significant antiproliferative activity in preclinical melanoma studies. ${ }^{74}$ Currently, a number of active molecules targeting MEK are under investigation, and have shown promising results in preclinical and clinical trials.

GSK1120212 (GlaxoSmithKline, Brentford, UK) is a potent and selective allosteric inhibitor of MEK1/2. A Phase I/II clinical trial including 20 evaluable patients with a $B-R A F$ mutant melanoma treated with $2 \mathrm{mg}$ of daily oral dose of GSK1120212 (the recommended Phase II dose) showed an RR of $40 \%$ and SD in $18 \%$. The therapy was well tolerated. ${ }^{75}$ The most common adverse events were an acneiform rash (85\%), diarrhea (48\%), fatigue (37\%), nausea (20\%), and vomiting (24\%). The ocular toxicities, an uncommon class effect, include central serous retinopathy and retinal vein occlusion, seen in three and one patient out of 162, respectively. A two-arm, open-label, randomized Phase III study comparing single-agent GSK1120212 to chemotherapy (either DTIC or paclitaxel [Bristol-Myers Squibb, Princeton, NJ]) in patients with unresectable melanoma harboring V600E mutations has recently been completed and results are awaited (NCT01245062).

Selumetinib (AZD6244, Array BioPharma, Boulder, CO), a selective MEK inhibitor, was compared with temozolamide in a randomized Phase II study for metastatic or locally advanced melanoma. At $100 \mathrm{mg}$ twice-daily oral dose, there was no significant difference noted between two arms in terms of the primary end point, PFS. In a subset of patients with tumors harboring B-RAF ${ }^{\mathrm{V} 600 \mathrm{E}}$ mutations (45 patients out of 200), five patients had an objective response $(11 \%) .^{76}$ Several other MEK inhibitors are currently being investigated in early clinical trials.

\section{Combinations of B-RAF and MEK inhibitors}

The majority of the patients treated with B-RAF inhibitors eventually develop disease progression. For example, the median PFS for vemurafenib in the BRIM-3 trial was 5.5 months. Various mechanisms of acquired resistance to vemurafenib have been described. Resistance to B-RAF inhibition can be mediated by several different mechanisms that restore ERK activation. This may occur upstream (new N-RAS mutations, up regulation of $\mathrm{C}-\mathrm{RAF}$, and upregulation of receptor tyrosine kinases, PDGFRB, ERBB2), or downstream (such as new activating MEK mutations or activation of serine/threonine MAPK kinases [COT] or in parallel signaling pathways, particularly the PI3K-AKT pathway). ${ }^{77-80}$ Consequently, MEK inhibition in addition to B-RAF inhibition can potentially overcome the resistance mediated by mechanisms upstream of B-RAF. A Phase I/II clinical trial combining GSK436 (GlaxoSmithKline, Brentford, UK) (B-RAF inhibitor) and GSK212 (GlaxoSmithKline, Brentford, UK) (MEK inhibitor) demonstrated tolerability and dramatic clinical activity. In the cohort treated with the maximum tolerated dose combination (GSK436 $200 \mathrm{mg}$ daily and GSK212 $1.5 \mathrm{mg}$ daily, both given orally), of a total of 19 patients, the RR was $74 \%$ (CR in four and PR in ten patients), and SD was seen in five patients. ${ }^{81}$ Interestingly, the B-RAF and MEK inhibitor combination appears to be associated with a lower incidence of skin toxicities: fewer rashes were seen (25\%) and only one case of cutaneous squamous cell carcinoma was seen in the whole Phase I/II cohort (109 patients).

Another combination of a B-RAF inhibitor (RAF265 [Chiron Corp, Emeryville, CA]) and an MEK inhibitor (MEK162) is currently being tested in advanced solid tumors harboring RAS and B-RAF ${ }^{\mathrm{V} 600 \mathrm{E}}$ mutations (NCT01352273).

\section{PTEN loss and use of PI3K inhibitors in melanoma}

Constitutive activation of the AKT/PI3K pathway has been implicated in many preclinical and clinical melanoma studies. ${ }^{23}$ AKT has been shown to be overexpressed in nearly $60 \%$ of all melanomas. ${ }^{82}$ Significant decreases in PTEN expression occur in $43 \%$ of melanoma tumors and are associated with aggressive tumor behavior. ${ }^{83}$ ERK-dependent upregulation of c-Jun leads to suppression of PTEN expression and concomitant activation of the PI3K/AKT/mTOR pathway. Therefore, targeting the PI3K pathway appears to be a promising approach in melanoma. A 
Phase I clinical trial combining PI3K inhibitors with MEK inhibitors (PI3K inhibitor BAY80-6946 [Bayer Healthcare Pharmaceuticals, Leverkusen, Germany] [intravenous] and MEK inhibitor BAY86-9766 [Bayer Healthcare Pharmaceuticals, Leverkusen, Germany] [oral]; NCT01392521) is currently enrolling patients. It remains to be determined if loss of PTEN correlates with activity of PI3K and whether it can be used as a predictive biomarker of PI3K treatment.

\section{Current status and future directions for personalized therapy for metastatic melanoma}

At present, vemurafenib is the only approved therapy that specifically targets an aberrant molecule in melanocytes. However the field is rapidly expanding, and many newer molecules are likely to be approved in the near future. $B-R A F$ mutation testing is now routinely done in patients with newly diagnosed metastatic melanoma. However, the decision about starting treatment with a B-RAF inhibitor is based on the individual clinical scenario, and many patients whose tumors harbor V600E mutations may initiate treatment with other therapies such as ipilimumab or IL-2. Vemurafenib can be helpful for patients who are symptomatic and require a rapid response. Immune-based therapies are associated with less frequent and sometimes slower responses; however, these responses are often durable and can result in prolonged survival. Predictive biomarkers for immune-based therapies are still lacking. Furthermore, biomarkers predictive of a short duration of response to melanocyte-targeted therapies are similarly lacking and are the subject of intense research. Although progress has been made in personalized therapy for melanoma in recent years, the next decade will likely bring additional therapies with improved companion diagnostics.

\section{Disclosure}

Harriet Kluger has served as a consultant to Genentech. The other authors have no conflicts of interest to declare for this work.

\section{References}

1. Siegel R, Naishadham D, Jemal A. Cancer statistics, 2012. CA Cancer J Clin. 2012;62(1):10-29.

2. Balch CM, Gershenwald JE, Soong SJ, et al. Final version of 2009 AJCC melanoma staging and classification. J Clin Oncol. 2009;27(36): 6199-6206

3. Atkins MB, Lotze MT, Dutcher JP, et al. High-dose recombinant interleukin 2 therapy for patients with metastatic melanoma: analysis of 270 patients treated between 1985 and 1993. J Clin Oncol. 1999;17(7): 2105-2116.
4. Middleton MR, Grob JJ, Aaronson N, et al. Randomized phase III study of temozolomide versus dacarbazine in the treatment of patients with advanced metastatic malignant melanoma. J Clin Oncol. 2000;18(1): 158-166.

5. Sznol M, Powderly D, Smith DC, et al. Safety and antitumor activity of biweekly MDX-1106 (Anti-PD-1, BMS-936558/ONO-4538) in patients with advanced refractory malignancies [abstract 2506]. J Clin Oncol. 2010;28 Suppl:15s.

6. Atkins MB, Kunkel L, Sznol M, Rosenberg SA. High-dose recombinant interleukin-2 therapy in patients with metastatic melanoma: long-term survival update. Cancer J Sci Am. 2000;6 Suppl 1:S11-S14.

7. Besser MJ, Treves AJ, Itzhaki O, et al. Adoptive cell therapy for metastatic melanoma patients: pre-clinical development at the Sheba Medical Center. Isr Med Assoc J. 2006;8(3):164-168.

8. Dudley ME, Yang JC, Sherry R, et al. Adoptive cell therapy for patients with metastatic melanoma: evaluation of intensive myeloablative chemoradiation preparative regimens. J Clin Oncol. 2008;26(32): 5233-5239.

9. Hodi FS, O'Day SJ, McDermott DF, et al. Improved survival with ipilimumab in patients with metastatic melanoma. $N$ Engl J Med. 2010; 363(8):711-723.

10. Robert C, Thomas L, Bondarenko I, et al. Ipilimumab plus dacarbazine for previously untreated metastatic melanoma. N Engl J Med. 2011; 364(26):2517-2526.

11. Sznol M. Molecular markers of response to treatment for melanoma. Cancer J. 2011;17(2):127-133.

12. Hamid O, Tsuchihashi Z, Alaparthy S, Galbraith S, Berman D. Association of baseline and on-study tumor biopsy markers with clinical activity in patients (pts) with advanced melanoma treated with ipilimumab [abstract 9008]. J Clin Oncol. 2009;27 Suppl:15S.

13. Hamid O, Schmidt H, Nissan A, et al. A prospective phase II trial exploring the association between tumor microenvironment biomarkers and clinical activity of ipilimumab in advanced melanoma. $J$ Transl Med. 2011;9:204.

14. Yuan J, Adamow M, Ginsberg BA, et al. Integrated NY-ESO-1 antibody and $\mathrm{CD} 8^{+} \mathrm{T}$-cell responses correlate with clinical benefit in advanced melanoma patients treated with ipilimumab. Proc Natl Acad Sci U SA. 2011;108(40):16723-16728.

15. Goff SL, Robbins PF, El-Gamil M, Rosenberg SA. No correlation between clinical response to CTLA-4 blockade and presence of NYESO-1 antibody in patients with metastatic melanoma. J Immunother. 2009;32(8):884-885.

16. Tarhini AA, Edington H, Butterfield LH, et al. Neoadjuvant ipilimumab in patients with stage IIIB/C melanoma: immunogenicity and biomarker analysis [abstract 8536]. J Clin Oncol. 2011;29 Suppl:15S.

17. Hodi FS. Tissue and blood biomarkers from patients with stage iii or stage iv melanoma treated with ipilimumab with or without sargramostim. Available from: http://clinicaltrials.gov/ct2/show/ NCT01489423. Accessed July 14, 2012.

18. Topalian SL, Hodi FS, Brahmer JR, et al. Safety, activity, and immune correlates of anti-PD-1 antibody in cancer. New Engl J Med. 2012; 366(26):2443-2454.

19. Brahmer JR, Tykodi SS, Chow LQ, et al. Safety and activity of antiPD-L1 antibody in patients with advanced cancer. New Engl J Med. 2012;366(26):2455-2465.

20. Iwai Y, Ishida M, Tanaka Y, Okazaki T, Honjo T, Minato N. Involvement of PD-L1 on tumor cells in the escape from host immune system and tumor immunotherapy by PD-L1 blockade. Proc Natl Acad Sci U S A. 2002;99(19):12293-12297.

21. Topalian SL, Drake CG, Pardoll DM. Targeting the PD-1/ B7-H1(PD-L1) pathway to activate anti-tumor immunity. Curr Opin Immunol. 2012;24(2):207-212.

22. Satyamoorthy K, Li G, Gerrero MR, et al. Constitutive mitogenactivated protein kinase activation in melanoma is mediated by both BRAF mutations and autocrine growth factor stimulation. Cancer Res. 2003;63(4):756-759. 
23. Dhawan P, Richmond A. A novel NF-kappa B-inducing kinase-MAPK signaling pathway up-regulates NF-kappa B activity in melanoma cells. J Biol Chem. 2002;277(10):7920-7928.

24. Liu J, Suresh Kumar KG, Yu D, et al. Oncogenic BRAF regulates betaTrcp expression and NF-kappaB activity in human melanoma cells. Oncogene. 2007;26(13):1954-1958.

25. Brose MS, Volpe P, Feldman M, et al. BRAF and RAS mutations in human lung cancer and melanoma. Cancer Res. 2002;62(23):6997-7000.

26. Dhomen N, Marais R. BRAF signaling and targeted therapies in melanoma. Hematol Oncol Clin North Am. 2009;23(3):529-545, ix.

27. Rubinstein JC, Sznol M, Pavlick AC, et al. Incidence of the V600K mutation among melanoma patients with BRAF mutations, and potential therapeutic response to the specific BRAF inhibitor PLX4032. J Transl Med. 2010;8:67.

28. Pollock PM, Cohen-Solal K, Sood R, et al. Melanoma mouse model implicates metabotropic glutamate signaling in melanocytic neoplasia. Nat Genet. 2003;34(1):108-112.

29. Sharma A, Trivedi NR, Zimmerman MA, Tuveson DA, Smith CD, Robertson GP. Mutant V599EB-Raf regulates growth and vascular development of malignant melanoma tumors. Cancer Res. 2005; 65(6):2412-2421.

30. Furge KA, Kiewlich D, Le P, et al. Suppression of Ras-mediated tumorigenicity and metastasis through inhibition of the Met receptor tyrosine kinase. Proc Natl Acad Sci U S A. 2001;98(19):10722-10727.

31. Bardeesy N, Kim M, Xu J, et al. Role of epidermal growth factor receptor signaling in RAS-driven melanoma. Mol Cell Biol. 2005; 25(10):4176-4188.

32. Jakob JA, Bassett RL Jr, Ng CS, et al. NRAS mutation status is an independent prognostic factor in metastatic melanoma. Cancer. Epub December 16, 2011.

33. Devitt B, Liu W, Salemi R, et al. Clinical outcome and pathological features associated with NRAS mutation in cutaneous melanoma. Pigment Cell Melanoma Res. 2011;24(4):666-672.

34. Monsel G, Ortonne N, Bagot M, Bensussan A, Dumaz N. c-Kit mutants require hypoxia-inducible factor 1alpha to transform melanocytes. Oncogene. 2010;29(2):227-236.

35. Poynter JN, Elder JT, Fullen DR, et al. BRAF and NRAS mutations in melanoma and melanocytic nevi. Melanoma Res. 2006;16(4): 267-273.

36. Long GV, Menzies AM, Nagrial AM, et al. Prognostic and clinicopathologic associations of oncogenic BRAF in metastatic melanoma. J Clin Oncol. 2011;29(10):1239-1246.

37. Joseph RW, Sullivan RJ, Harrell R, et al. Correlation of NRAS mutations with clinical response to high-dose IL-2 in patients with advanced melanoma. J Immunother. 2012;35(1):66-72.

38. Van Raamsdonk CD, Griewank KG, Crosby MB, et al. Mutations in GNA11 in uveal melanoma. $N$ Engl J Med. 2010;363(23): 2191-2199.

39. Besaratinia A, Pfeifer GP. Uveal melanoma and GNA11 mutations: a new piece added to the puzzle. Pigment Cell Melanoma Res. 2011; 24(1):18-20.

40. Van Raamsdonk CD, Bezrookove V, Green G, et al. Frequent somatic mutations of GNAQ in uveal melanoma and blue naevi. Nature. 2009; 457(7229):599-602.

41. Yarden Y, Kuang WJ, Yang-Feng T, et al. Human proto-oncogene c-kit: a new cell surface receptor tyrosine kinase for an unidentified ligand. EMBO J. 1987;6(11):3341-3351.

42. McNiece IK, Briddell RA. Stem cell factor. J Leukoc Biol. 1995;58(1): $14-22$

43. Lux ML, Rubin BP, Biase TL, et al. KIT extracellular and kinase domain mutations in gastrointestinal stromal tumors. Am J Pathol. 2000;156(3): 791-795.

44. Carvajal RD, Antonescu CR, Wolchok JD, et al. KIT as a therapeutic target in metastatic melanoma. JAMA. 2011;305(22):2327-2334.

45. Curtin JA, Busam K, Pinkel D, Bastian BC. Somatic activation of KIT in distinct subtypes of melanoma. J Clin Oncol. 2006;24(26):4340-4346.
46. Garrido MC, Bastian BC. KIT as a therapeutic target in melanoma. J Invest Dermatol. 2010;130(1):20-27.

47. Tsai J, Lee JT, Wang W, et al. Discovery of a selective inhibitor of oncogenic B-Raf kinase with potent antimelanoma activity. Proc Natl Acad Sci U S A. 2008;105(8):3041-3046.

48. Flaherty KT, Puzanov I, Kim KB, et al. Inhibition of mutated, activated BRAF in metastatic melanoma. New Engl J Med. 2010;363(9): 809-819.

49. Sosman JA, Kim KB, Schuchter L, et al. Survival in BRAF V600-mutant advanced melanoma treated with vemurafenib. New Engl J Med. 2012;366(8):707-714

50. Chapman PB, Hauschild A, Robert C, et al. Improved survival with vemurafenib in melanoma with BRAF V600E mutation. New Engl J Med. 2011;364(26):2507-2516.

51. Kefford R, Arkenau H, Brown MP, et al. Phase I/II study of GSK2118436, a selective inhibitor of oncogenic mutant BRAF kinase, in patients with metastatic melanoma and other solid tumors [abstract 8503]. J Clin Oncol. 2010;28 Suppl:15s.

52. Su F, Viros A, Milagre C, et al. RAS mutations in cutaneous squamouscell carcinomas in patients treated with BRAF inhibitors. New Engl J Med. 2012;366(3):207-215.

53. Halait H, Demartin K, Shah S, et al. Analytical performance of a realtime PCR-based assay for V600 mutations in the BRAF gene, used as the companion diagnostic test for the novel BRAF inhibitor vemurafenib in metastatic melanoma. Diagn Mol Pathol. 2012;21(1):1-8.

54. Tan YH, Liu Y, Eu KW, et al. Detection of BRAF V600E mutation by pyrosequencing. Pathology. 2008;40(3):295-298.

55. Anderson S, Bloom KJ, Vallera DU, et al. Multisite analytic performance studies of a real-time polymerase chain reaction assay for the detection of BRAF V600E mutations in formalin-fixed paraffinembedded tissue specimens of malignant melanoma. Arch Pathol Lab Med. Epub February 14, 2012.

56. Capper D, Berghoff AS, Magerle M, et al. Immunohistochemical testing of BRAF V600E status in 1,120 tumor tissue samples of patients with brain metastases. Acta Neuropathol. 2012;123(2):223-233.

57. Andrulis M, Penzel R, Weichert W, von Deimling A, Capper D. Application of a BRAF V600E mutation-specific antibody for the diagnosis of hairy cell leukemia. Am J Surg Pathol. Epub April 22, 2012.

58. Demetri GD, von Mehren M, Blanke CD, et al. Efficacy and safety of imatinib mesylate in advanced gastrointestinal stromal tumors. New Engl J Med. 2002;347(7):472-480.

59. Druker BJ, Talpaz M, Resta DJ, et al. Efficacy and safety of a specific inhibitor of the BCR-ABL tyrosine kinase in chronic myeloid leukemia. N Engl J Med. 2001;344(14):1031-1037.

60. Shen SS, Zhang PS, Eton O, Prieto VG. Analysis of protein tyrosine kinase expression in melanocytic lesions by tissue array. J Cutan Pathol. 2003;30(9):539-547.

61. Ugurel S, Hildenbrand R, Zimpfer A, et al. Lack of clinical efficacy of imatinib in metastatic melanoma. Br J Cancer. 2005;92(8): 1398-1405.

62. Wyman K, Atkins MB, Prieto V, et al. Multicenter phase II trial of high-dose imatinib mesylate in metastatic melanoma: significant toxicity with no clinical efficacy. Cancer. 2006;106(9):2005-2011.

63. Kim KB, Eton O, Davis DW, et al. Phase II trial of imatinib mesylate in patients with metastatic melanoma. Br J Cancer. September 2 , 2008;99(5):734-740.

64. Hodi FS, Friedlander P, Corless CL, et al. Major response to imatinib mesylate in KIT-mutated melanoma. J Clin Oncol. April 20, 2008;26(12):2046-2051.

65. Lutzky J, Bauer J, Bastian BC. Dose-dependent, complete response to imatinib of a metastatic mucosal melanoma with a K642E KIT mutation. Pigment Cell Melanoma Res. Aug 2008;21(4):492-493.

66. Satzger I, Kuttler U, Volker B, Schenck F, Kapp A, Gutzmer R. Anal mucosal melanoma with KIT-activating mutation and response to imatinib therapy - case report and review of the literature. Dermatology. 2010;220(1):77-81. 
67. Yamaguchi M, Harada K, Ando N, Kawamura T, Shibagaki N, Shimada S. Marked response to imatinib mesylate in metastatic acral lentiginous melanoma on the thumb. Clin Exp Dermatol. Mar 2011;36(2): 174-177.

68. Guo J, Si L, Kong Y, et al. Phase II, open-label, single-arm trial of imatinib mesylate in patients with metastatic melanoma harboring c-Kit mutation or amplification. J Clin Oncol. July 20, 2011;29(21): 2904-2909.

69. Cho JH, Kim KM, Kwon M, Kim JH, Lee J. Nilotinib in patients with metastatic melanoma harboring KIT gene aberration. Invest New Drugs. November 9, 2011.

70. Kluger HM, Dudek AZ, McCann C, et al. A phase 2 trial of dasatinib in advanced melanoma. Cancer. May 15, 2011;117(10):2202-2208.

71. Minor DR, Kashani-Sabet M, Garrido M, O’Day SJ, Hamid O, Bastian BC. Sunitinib therapy for melanoma patients with KIT mutations. Clin Cancer Res. March 1, 2012;18(5):1457-1463.

72. Quintas-Cardama A, Lazar AJ, Woodman SE, Kim K, Ross M, Hwu P. Complete response of stage IV anal mucosal melanoma expressing KIT Val560Asp to the multikinase inhibitor sorafenib. Nat Clin Pract Oncol. Dec 2008;5(12):737-740.

73. Woodman SE, Trent JC, Stemke-Hale K, et al. Activity of dasatinib against L576P KIT mutant melanoma: molecular, cellular, and clinical correlates. Mol Cancer Ther. Aug 2009;8(8):2079-2085.

74. Zhang XD, Borrow JM, Zhang XY, Nguyen T, Hersey P. Activation of ERK $1 / 2$ protects melanoma cells from TRAIL-induced apoptosis by inhibiting Smac/DIABLO release from mitochondria. Oncogene. May 15, 2003;22(19):2869-2881.

75. Infante JR, Nallapareddy S, Gordon MS, et al. Messersmith. Safety and efficacy results from the first-in-human study of the oral MEK $1 / 2$ inhibitor GSK1120212. Paper presented at: ASCO annual meeting 2010 .
76. Dummer R, Chapman PB, Sosman JA, et al. AZD6244 (ARRY-142886) vs temozolomide (TMZ) in patients (pts) with advanced melanoma: An open-label, randomized, multicenter, phase II study. Paper presented at: ASCO annual meeting 2008; Chicago, IL, USA.

77. Nazarian R, Shi H, Wang Q, et al. Melanomas acquire resistance to B-RAF(V600E) inhibition by RTK or N-RAS upregulation. Nature. December 16, 2010;468(7326):973-977.

78. Johannessen CM, Boehm JS, Kim SY, et al. COT drives resistance to RAF inhibition through MAP kinase pathway reactivation. Nature. December 16, 2010;468(7326):968-972.

79. Montagut C, Sharma SV, Shioda T, et al. Elevated CRAF as a potential mechanism of acquired resistance to BRAF inhibition in melanoma. Cancer Research. June 15, 2008;68(12):4853-4861.

80. Wang H, Daouti S, Li WH, et al. Identification of the MEK1(F129L) activating mutation as a potential mechanism of acquired resistance to MEK inhibition in human cancers carrying the B-RafV600E mutation. Cancer research. August 15, 2011;71(16):5535-5545.

81. Infante JR, Lawrence DP, Weber JS, et al. Phase I/II study to assess safety, pharmacokinetics, and efficacy of the oral MEK 1/2 inhibitor GSK1120212 (GSK212) dosed in combination with the oral BRAF inhibitor GSK2118436 (GSK436). Paper presented at: ASCO annual meeting 2011; Chicago, IL, USA.

82. Stahl JM, Sharma A, Cheung M, et al. Deregulated Akt3 activity promotes development of malignant melanoma. Cancer research. October 1, 2004;64(19):7002-7010.

83. Mikhail M, Velazquez E, Shapiro R, et al. PTEN expression in melanoma: relationship with patient survival, Bcl-2 expression, and proliferation. Clinical Cancer Research: An Official Journal of the American Association for Cancer Research. July 15, 2005;11(14):5153-5157.
Pharmacogenomics and Personalized Medicine

\section{Publish your work in this journal}

Pharmacogenomics and Personalized Medicine is an international, peerreviewed, open access journal characterizing the influence of genotype on pharmacology leading to the development of personalized treatment programs and individualized drug selection for improved safety, efficacy and sustainability. This journal is indexed on the American Chemical

\section{Dovepress}

Society's Chemical Abstracts Service (CAS). The manuscript management system is completely online and includes a very quick and fair peer-review system, which is all easy to use. Visit http://www.dovepress. com/testimonials.php to read real quotes from published authors. 